\title{
Advances in Multispectral and Hyperspectral Imaging for Archaeology and Art Conservation
}

\author{
Haida Liang \\ School of Science and Technology, Nottingham Trent University, Clifton Lane, \\ Nottingham NG11 8NS, UK e-mail: haida.liang@ntu.ac.uk
}

The date of receipt and acceptance will be inserted by the editor

\begin{abstract}
Multispectral imaging has been applied to the field of art conservation and art history since the early 1990s. It is attractive as a noninvasive imaging technique because it is fast and hence capable of imaging large areas of an object giving both spatial and spectral information. This paper gives an overview of the different instrumental designs, image processing techniques and various applications of multispectral and hyperspectral imaging to art conservation, art history and archaeology. Recent advances in the development of remote and versatile multispectral and hyperspectral imaging as well as techniques in pigment identification will be presented. Future prospects including combination of spectral imaging with other noninvasive imaging and analytical techniques will be discussed.
\end{abstract}

Key words multispectral imaging - hyperspectral imaging - pigment identification - spectral imaging - imaging spectroscopy - AOTF - LCTF remote imaging - infrared imaging - colour - non-invasive imaging - XRF - laser scanning - optical coherence tomograghy

\section{Introduction}

Multispectral and hyperspectral imaging collect images of an object in a series of spectral windows. They are efficient methods for collecting millions of spectra since a spectrum is measured for each spatial pixel (Fig. 1). The distinction between multispectral and hyperspectral imaging is rather blurred and very much discipline dependent. In general, hyperspectral imaging consists of more finely divided spectral channels than multispectral imaging. Multispectral imaging can sometimes refer to a set of images taken at vastly

Send offprint requests to: Haida Liang 


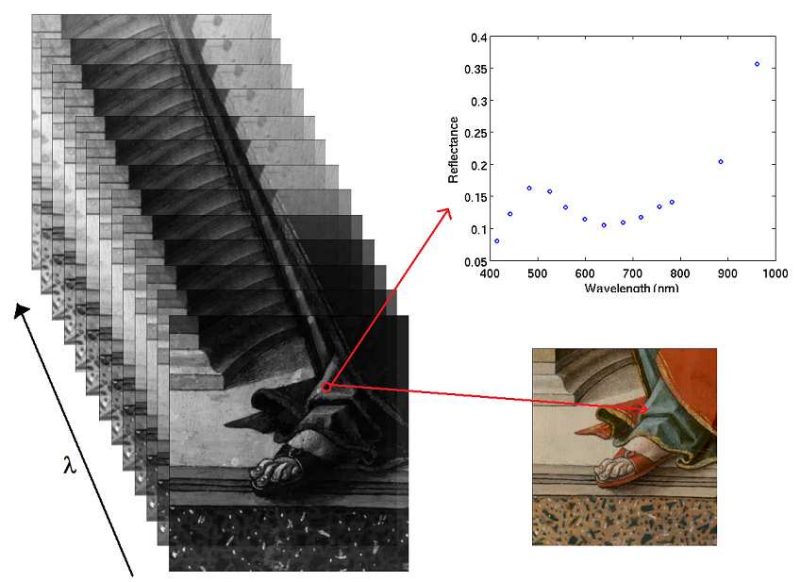

Fig. 1 A schematic diagram illustrating a spectral cube obtained from multispectral imaging, the colour image derived from the spectral cube and a spectrum for a point on the blue colour which can be identified with the pigment azurite.

different parts of the electromagnetic spectrum, e.g. three visible images in red, blue and green, an infrared image and an X-ray image of an object [1] [2]. We will not use this definite of multispectral imaging here. For the rest of the paper, we will refer to multispectral and hyperspectral imaging together as spectral imaging or imaging spectroscopy.

Multispectral and hyperspectral imaging were first developed for remote sensing, which can include planetary science and astronomy [3]. More recently, spectral imaging has found applications in terrestrial laboratories for applications in biology, medicine, chemistry, industrial sorting, quality control and surveillance [4] [5]. Multispectral imaging has been applied to the field of cultural heritage since the early 1990s. As a non-invasive imaging technique, it has the advantage over invasive techniques in that investigations can be carried out on any object (even on intact and fragile ones where samples cannot be taken) and anywhere on an object. It was first applied for qualitative band to band comparison in order to identify areas of different material composition, natural degradation of material, past conservation intervention, preparatory sketches, and quantitatively for improved precision in colour measurement. Later, with increased number of bands and speed of acquisition, it was used to extract spectral reflectance information for pigment identification. It can operate in the UV, visible, the near infrared and in UV-fluorescence mode.

Multispectral and hyperspectral imaging are in increasing demand in the field of art conservation, art history and archaeology judging by the number of recent reviews on the subject from the conservation and archaeology community [6] [7].

Spectral imaging has mostly been applied to paintings and manuscripts. In the case of paintings, multispectral imaging was first developed to in- 
crease the colour fidelity of the images. In the past 20 years, a number of EU projects has been dedicated to the design and implementation of high colour fidelity, high resolution scanning systems for the recording of museum paintings and other objects of art (e.g. [8] [9] [10] [11] [12] [13] [56]).

\section{Instrument Design Considerations}

A spectral imaging system needs the following essential components: lighting, focusing optics, detector and most importantly a means of wavelength selection. There is a diverse range of methods for wavelength selection which determines the design of the illumination system and the spatial and spectral scanning strategy.

\subsection{Illumination requirements}

One of the special requirements for imaging of heritage objects is minimum light exposure to ensure that light-induced ageing of the objects is kept at a minimum. There is a vast body of work done on light-induced ageing which has informed display strategies in museums (e.g. [14]). In general, UV and thermal radiation are eliminated from a white light source before it is used to illuminate objects in museums. For example, for oil paintings the recommended level is $\sim 200$ lux and for manuscripts and other paper-based artworks the lighting level is kept at $\sim 50$ lux. It is generally assumed that the reciprocity principle holds, which basically says that light-induced damage is determined by the accumulated total energy incident on a material rather than the intensity of the incident light. The reciprocity principle has been widely used to justify high intensity illumination for fast imaging (e.g. [11]). To achieve the same signal-to-noise ratio in an image, one can either illuminate the object with a low intensity light for longer or a high intensity light for a shorter period of time. Since it is the total energy incident on an object that determines the damage, it appears sensible to use a higher intensity light to increase the imaging efficiency without causing extra damage. However, extra caution must be exercised at high intensity levels since the reciprocity principle must break down at some level. Saunders et al. [15] conducted a survey of 21 light sensitive pigments and found that they all follow the reciprocity principle up to $8 \times 10^{3}$ lux. Our recent studies using a microfading spectrometer [16] have found that some light sensitive pigments such as orpiment, where the light induced degradation is due to competing reactions, reciprocity does not hold [17]. It was found that for these very sensitive pigments illuminated with $2 \times 10^{6}$ lux of incident light, it takes $\sim 30$ seconds illumination for any measurable damage to occur and minutes for any damage that is noticeable by the naked eye. The rate of degradation is always greatest within the first $\sim 30$ seconds. Therefore, care must be taken to choose the right illumination level. It might be sensible to do spot tests for light sensitive material using microfading spectrometry prior to high intensity illumination. 


\subsection{Wavelength Selection}

Wavelength selection can be achieved either on the illumination light path such that only a selected wavelength range of light is incident on the object at a time, or on the reflected light path before the detector such that the light reflected from the object can be separated spectrally.

2.2.1 Wavelength selection through illumination The first multispectral imaging system designed for paintings was through filtering the illumination and using a monochrome digital camera to collect the reflected light [9]. Interference filters were placed in front of a halogen-tungsten light source. The advantage of such a system is economic light exposure since only a narrow wavelength range is incident on the object at a time. This is very important for light sensitive materials especially paper-based works of art or manuscripts. The requirements for the filtering system are high throughput and low out-of-band response within the detector sensitive wavelength range, and good thermal stability as they are placed close to the light source. The other advantage is that any off-the-shelf monochrome camera and lens system can be used without modification. However, in practical terms an average camera lens has significant chromatic aberration such that images collected at widely different wavelengths will have different focal length resulting in significant difference in magnification [18] which will then need to be corrected in a post-processing software. While such a system is compatible with a wide range of cameras, it can be less flexible and portable in terms of the lighting component of the system. The contribution from background light can be significant when there is no spectral filtering in front of the camera, which restricts the use of such systems outside studios. With the development of luminous LEDs, this option is becoming more attractive since LEDs are energy efficient [19]. One of the potential disadvantages of such a system is that they cannot be used for UV-fluorescence imaging because there is no spectral filtering in front of the detector.

2.2.2 Wavelength selection in the reflected light Alternatively, wavelength selection can be achieved through either filtering or dispersing the reflected light. In the snapshot mode, the entire spectrum is collected simultaneously per spatial point and a spatial area is collected through scanning in a time sequence. In the sequential mode, the spatial field of view is imaged through one wavelength channel simultaneously and the full spectral cube is collected through sequential spectral filtering. The snapshot mode has its advantage when imaging objects that are time varying over a time scale shorter than the imaging time. These can be of concern in remote sensing, astronomy or biomedical imaging where there are time dependent signals. In imaging of cultural artefacts, this is rarely a problem which is why only recently that imaging in the snapshot mode has been adopted.

2.2.3 Sequential spectral filtering Sequential spectral filtering usually involves placing the filtering system between the lens and the detector, which 
means that specialised lens may have to be used to adapt to the increased distance between the lens and the detector. Placing the filtering system in front of the lens is practically difficult because of the large aperture of the filtering system required to avoid vignetting. Filtering systems with such a large aperture is either costly or not available.

The simplest method for wavelength selection is to place bandpass interference filters between the lens and the detector [20] [12] [13] [21] [47]. Filter selection can be computer controlled through a motorised filter wheel. Custom designed filter sets for imaging purposes that have the same optical thickness will ensure that the image scale stay the same for different wavelengths. Such a filter set combined with a high quality lens designed for minimum chromatic aberration ensures that no image scaling is necessary between channels [13]. However, a tilt of the position of the filter by a fraction of a degree can result in noticeable shifts in the image. As will be discussed in Sec. 4, image shifting and re-scaling can easily be dealt with automatically in post-processing. The advantage of interference filters is large aperture, large field of view, good optical quality and low cost. The disadvantage is the fixed number of filters and slow speed in filter change since it takes time for the filter wheel to move and settle into a new position. Linear/circular variable interference filters can also be used to mechanically move through the wavelength channels.

Tunable filters such as Liquid Crystal Tunable Filters (LCTF) and AcoustoOptical Tunable Filters (AOTF) offer rapid tuning between wavelength channels and have no moving parts. Both offer random access for wavelength selection. By taking care in the optical design, it is possible to produce high quality images that are not shifted between wavelength channels.

LCTF can be tuned by changing the voltage applied to a birefringent liquid crystal. It is essentially an electrically tuned Lyot filter. The tuning speed is given by the relaxation time of the crystal and is typically of the order of tens to hundreds of milliseconds. The bandwidths of the filters are fixed and increases with central wavelength. The transmission efficiency decreases with decreasing wavelength resulting in relatively low blue response [22]. LCTFs are polarisation sensitive which means that the maximum overall throughput is less than $50 \%$. Hyperspectral imaging using LCTF has been explored for heritage applications in the $400 \mathrm{~nm}-700 \mathrm{~nm}$ range [23] [24] and the $650 \mathrm{~nm}-1040 \mathrm{~nm}$ range [25]. Since the reflectance spectra of pigments in the $400 \mathrm{~nm}$ to $1000 \mathrm{~nm}$ range is fairly smooth, the increased spectral resolution of a hyperspectral system (at the expense of decreased signal-to-noise ratio) did not provide much additional information.

AOTF is tuned by changing the frequency of acoustic or Radio Frequency (RF) waves applied to a birefringent crystal, typically $\mathrm{TeO}_{2}$ for wavelength range between $350 \mathrm{~nm}$ and $5000 \mathrm{~nm}$. The RF waves travelling across the crystal essentially set up a volume diffraction grating by producing a periodic modulation of the refractive index within the crystal through the photo-elastic effect [26]. AOTF is polarisation sensitive. The output from an AOTF consists of the undiffracted ray and the two orthogonally 
polarised diffracted beams angularly separated. There is a one-to-one correspondence between the RF frequency injected and the central wavelength of transmission. The bandwidth can also be tunable through injection of multiple RF frequencies [27]. The minimum achievable bandwidth also increases with wavelength. The speed of tuning is given by the travel time of the acoustic wave in the crystal and is typically of order of hundred microseconds [28]. While it is possible to recombine the orthogonally polarised diffracted beams to increase overall throughput, in practice it is difficult to maintain high optical quality through such combination.

Both LCTF and AOTF can tune up to an octave. AOTFs in general have higher throughput compared with LCTF and do not have a decreasing throughput with decreasing wavelength. On the other hand, LCTF have larger aperture and angular field of view. While AOTFs have faster tuning speed than LCTF, the tuning time in either case is insignificant compared with typical integration times and readout speed of an average $1000 \times 1000$ pixels CCD detector.

Other tunable devices include the Fabry-Pérot etalon and the Fourier transform Michelson interferometer. In the case of the Fabry-Pérot elaton, wavelength tuning is achieved through mechanically changing the distance between the mirrors using piezo-electric transducers [29] [30]. These devices have large aperture but small angular field of view. The Fourier transform Michelson imaging interferometer can also be used through scanning one of the two mirrors and collecting the two-dimensional interferogram on the detector. The Fast Fourier Transform (FFT) of the interference pattern of a pixel gives the reflectance spectrum. This is essentially performing FTIR spectroscopy for a number of spatial pixels simultaneously.

2.2.4 Simultaneous spectral collection One of the most common methods of spectral collection in remote sensing is to use a slit combined with a diffraction grating to disperse the light. A two-dimensional detector can collect a series of spectra corresponding to spatial points aligned with the slit simultaneously. A full 3D spectral cube can be collected by scanning spatially in the direction perpendicular to the slit [31] [32] [33]. Such devices can offer much higher spectral resolution than the tunable filters described above but with reduced flexibility. The full spectral range with a fixed spectral resolution is always collected. However, high spectral resolutions of less than $10 \mathrm{~nm}$ are generally not needed since spectral reflectance of pigments have fairly smooth spectral shape. Similarly, in remote sensing applications, it is well recognised that the maximum spectral resolution necessary is between $5 \mathrm{~nm}$ and 20nm [34].

Since the full spectral range is collected at once, it places high demands on the chromatic aberration tolerance of the lens. In the sequential filtering designs discussed previously, there is more flexibility in adjusting the focus and integration time for each individual spectral channel for best focus and optimum signal-to-noise ratio. 
Sagnac imaging interferometer is also used for the simultaneous spectral collection in a line of spatial pixels similar to the grating spectral imager, except a FFT is needed to recover the spectrum per pixel. Such a device has been used by Casini et al. [35] for UV-fluorescence imaging.

An alternative method of simultaneous wavelength collection has been used for multispectral imaging of paintings [36] [37] [38] where the reflected light from a single point is collected and distributed using a fibre bundle to a series of detectors with different interference filters. Such a device has the potential to reduce light scattering if only a small region around the point being measured is illuminated (similar to a fibre optic spectrometer setup). The drawback is the limited speed of capture.

Finally, another simple method of simultaneous spectra collection can be achieved by sacrificing spatial resolution of a CCD camera by having different filters placed in front of individual pixels similar to the concept of a RGB colour camera except more than 3 filters are involved (e.g. [39]). Such an instrument involves modifying the CCD sensor design which could be expensive. It is possible to recover the full spatial resolution by dithering the camera and taking multiple shots. The spectra measured simultaneously are not from exactly the same spot unlike previous methods. A hybrid design where a small number of interference filters are placed in front of a RGB colour camera rather than a monochrome camera has been applied to imaging paintings [40]. In this case there is no need for a special detector and a simple RGB camera can be used. The various combination of filters in front of the detector and on the detector pixels provides extra spectral resolution. However, the disadvantage is the attenuation of collected photons by going through two sets of filters as well as loss of spatial resolution.

\subsection{Detectors}

In the wavelength range between $300 \mathrm{~nm}-1000 \mathrm{~nm}$, silicon CCD detectors are used. For low noise detection, peltier cooled systems are preferred. In the Short Wave Infrared (SWIR) range between $900 \mathrm{~nm}-1700 \mathrm{~nm}$, mechanically cooled InGaAs detector arrays are the most commonly used due to their high sensitivity and low cost. While extended InGaAs can reach beyond $2 \mu \mathrm{m}$, they cannot compete with $\mathrm{HgCdTe}$ in sensitivity. $\mathrm{HgCdTe}$ detectors are sensitive to a broad wavelength range from $\sim 1 \mu \mathrm{m}$ to $\sim 10 \mu \mathrm{m}$. Currently, spectral imaging have mostly been conducted in the range between $400 \mathrm{~nm}$ and $1700 \mathrm{~nm}$, mainly because of the huge jump in cost for detector arrays that are sensitive beyond $1.7 \mu \mathrm{m}$. Some early works have pushed this up to $2.2 \mu \mathrm{m}$ using low sensitivity analogue detectors such as the $\mathrm{PbO}-\mathrm{PbS}$ vidicon. The only recent device used in art applications that managed to reach $2.3 \mu \mathrm{m}$ is the one that uses single element InGaAs detectors [38]. 


\section{Calibration and sources of error}

The method of calibrating multispectral or hyperspectral imaging involves the normal procedure of calibrating CCD images (e.g. [41]) as well as the calibration of the spectral response of the imaging system.

Thermal noise associated with CCD detectors include dark noise and readout noise [41]. The dark current and the noise associated with it can be reduced by using a cooled detector. The readout noise is also determined by the speed of the readout. The shot noise or photon noise is determined by the Poisson statistics of the quantum arrival of photons and is given by the square root of the number of photo-electrons detected.

Calibration of spectral images involves taking the following calibration images:

- dark images of the same integration time as the object frames but with the lens cap on (or illumination off) to correct for the accumulated thermal dark current;

- flatfield images per channel of a uniform white target to correct for the spatial inhomogeneity of illumination and pixel-to-pixel gain variation of the CCD;

- images of a spectral standard through each wavelength channel to correct for the spectral response of the imaging system.

The reflectance at a pixel (the $i$ th pixel) captured with a spectral channel of central wavelength $\lambda$ is given by

$$
R_{i}(\lambda)=R_{W}(\lambda) g \frac{\left(I_{i}(\lambda)-D_{i}(\lambda)\right) f_{i}(\lambda)}{\Sigma_{n}\left[\left(W_{i}(\lambda)-D W_{i}(\lambda)\right) f_{i}(\lambda)\right] / n}
$$

where $R_{W}$ is the true spectral reflectance of a spectral standard, $I_{i}$ is the counts for the light reflected from the object, $D_{i}$ is the dark counts for the same integration time as the object frame, $f_{i}$ is the flatfield correction factor for that pixel, $W_{i}$ is the counts for the spectral standard and $D W_{i}$ is the corresponding dark counts for the same integration time as the standard, $g$ is the scale factor to adjust the integration time of the object to that of the spectral standard and $n$ is the number of pixel over which to average the response of the spectral standard.

While CCD detectors are highly linear, non-linearity is still observed close to saturation and at very low exposure times. Optimum exposure time for maximum signal-to-noise is determined by the maximum counts over which the CCD is linear. For best quality images and spectral reflectance measurements, exposure times should be adjusted per channel for maximum signal-to-noise ratio images.

It is best to capture images after the light has been switched on for 10-20 minutes for a stable intensity of illumination and to avoid temperature gradients along the imaging pathlength which can degrade the spatial resolution of the system (similar to the 'seeing' effect in astronomy). 
The measured spectral reflectance of an object depends on the geometry of the illumination/collection setup which determines the relative amount of surface reflection to that of the diffuse reflection. For comparison between materials, it is best to image the objects in the same setup as the reference material. This is particularly important for systems with moderate spectral resolution.

Finally, the cross-talk due to scattered light (similar to the adjacency effect in remote sensing) can result in inaccuracy in the measured spectral reflectance. A dark area surrounded by bright regions would appear to be brighter than if it had been surrounded by dark regions, because of light scattered from the surrounding regions. Martinez et al. [57] showed that this scattering can be modelled as a linear function

$$
I_{m}(i)=I_{t}(i)+\alpha \bar{I}
$$

where $I_{m}$ is the measured intensity of a pixel, $I_{t}$ is the true intensity, $\bar{I}$ is the mean intensity of the image and $\alpha$ is the cross-talk scattering coefficient which can be measured by imaging a set of calibration targets with a neutral grey surrounded by white, grey and black backgrounds.

\section{Image alignment and mosaicing}

As mentioned above, for some instrument designs it is expected that the images from different spectral channels need to be shifted or even spatially re-scaled in order to have an aligned image cube. There are various algorithms associated with image registration (e.g. [42]). Image registrations involving only linear shifts are relatively simple and can be found by performing cross-correlation. Spatial image scaling involving re-sampling could result in some loss of information, therefore it is best to design the optics of the system to avoid scaling of the images.

High resolution imaging of large objects inevitably involves mosaicing of images. Adjacent images need to be taken with sufficient overlap to allow automatic image registration. Since the shifts are linear, simple crosscorrelation algorithm can be used for image registration [43] [44] [45].

\section{Applications}

\subsection{Qualitative inter-band comparisons for revealing hidden information}

Applications of spectral imaging in art conservation include the detection of damages and past intervention through inter-band comparisons. Figure 2 shows that the infrared spectral bands reveal areas of damage (black spots and a crack) that had been repaired and retouched such that they are invisible in the colour image and in many of the other visible spectral bands. Near infrared bands are particularly useful for this purpose and for revealing 


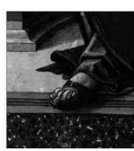

$400 \mathrm{~nm}$

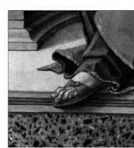

$600 \mathrm{~nm}$

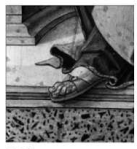

$800 \mathrm{~nm}$

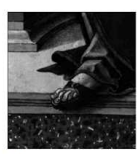

$440 \mathrm{~nm}$

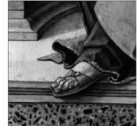

$640 \mathrm{~nm}$

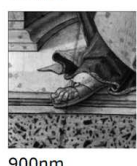

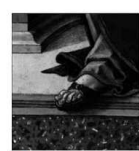

$480 \mathrm{~nm}$

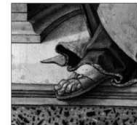

$680 \mathrm{~nm}$

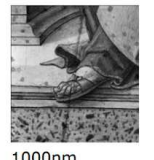

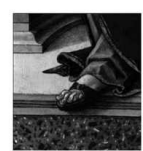

$520 \mathrm{~nm}$

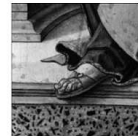

$720 \mathrm{~nm}$

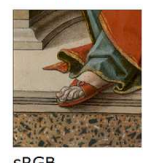

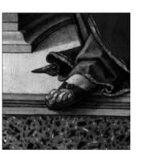

$560 \mathrm{~nm}$

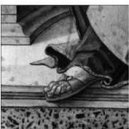

$760 \mathrm{~nm}$

Fig. 2 Multispectral images $(400-1100 \mathrm{~nm})$ taken with an upgraded VASARI system using the CRISATEL set of interference filters placed between the lens and the detector [13], and the derived colour image (see Sec. 5.2) rendered under the D65 illuminant of a region of a painting by Carlo Crivelli, Saint Catherine of Alexandria (NG 907.2) from the National Gallery, London collection.

the underdrawings (preparatory sketch) beneath the paint layers. Figure 3 shows that the $880 \mathrm{~nm}$ image reveals clearly the preparatory sketches beneath the paint layer. The sketches were drawn with a solid material and some of it were made by pouncing. Such information is invaluable to art historians in studying the techniques of painting and for attribution and authentication. Ever since the pioneering work of van Asperen de Boer in the 1960s [46] in developing a vidicon infrared camera for imaging underdrawings, it has been known that pigments are most transparent to infrared radiation in the $1 \mu \mathrm{m}$ to $2 \mu \mathrm{m}$ range. Earlier near infrared multispectral/hyperspectral imaging systems for the $1 \mu \mathrm{m}$ to $1.5 \mu \mathrm{m}$ were developed using infrared to optical converters or vidicon tube technology which is not linear and has low sensitivity [20] [47]. Nowadays InGaAs detectors are much more affordable and there are a number of SWIR spectral imaging systems developed for heritage applications [93][38][32].

Traditionally, UV-fluorescence imaging is considered to be the standard method for revealing erased or faded writing on manuscripts. However, it appears that other non-visible bands such as reflected light from the UV (rather than fluorescence) [50] and infrared bands [48] can also be effective at revealing 'hidden' writings. Figure 4 shows that a page of a prayer book that looked blank in the visible is revealed in the $880 \mathrm{~nm}$ channel to have a signature written on the top part of the page [48]. Multispectral imaging has been successfully used to separate the erased writing of Archimedes palimpsest from the later over-written text [49] and hyperspectral imaging has been shown to be effective in visual enhancement of old documents corrupted by ink bleed and ink corrosion [51]. 

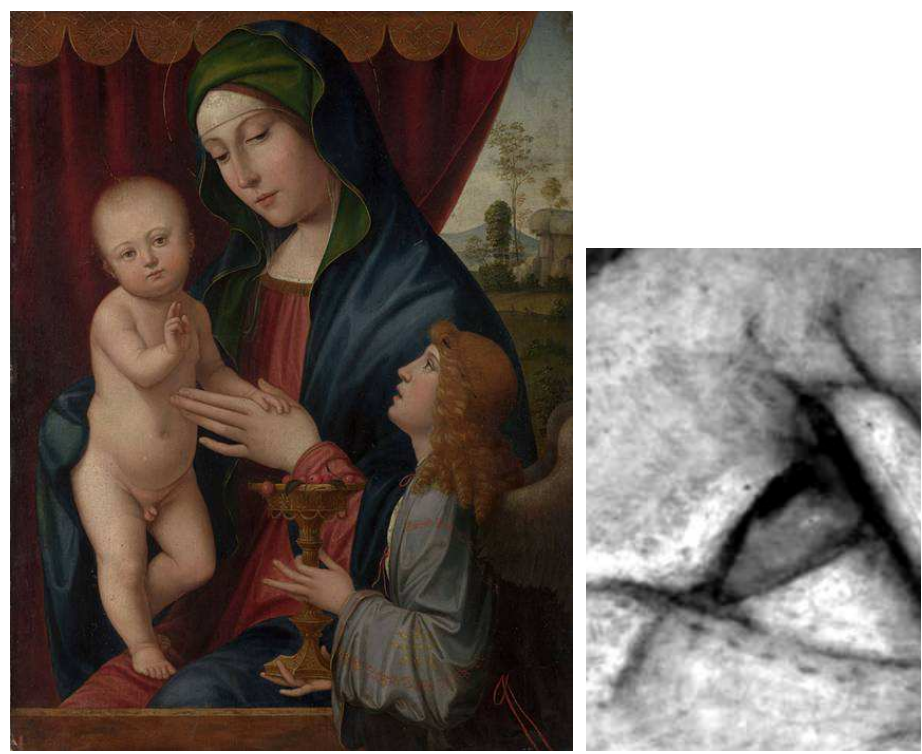

Fig. 3 Colour photo of a painting, The Virgin and Child with an Angel (NG 3927) after Francesco Francia (image copyright The National Gallery, London) and the $880 \mathrm{~nm}$ image of a region around the eye of the angel taken with PRISMS (see Sec. 6) [91]) at a distance of $6 \mathrm{~m}$ showing the preparatory drawings under the paint layer.
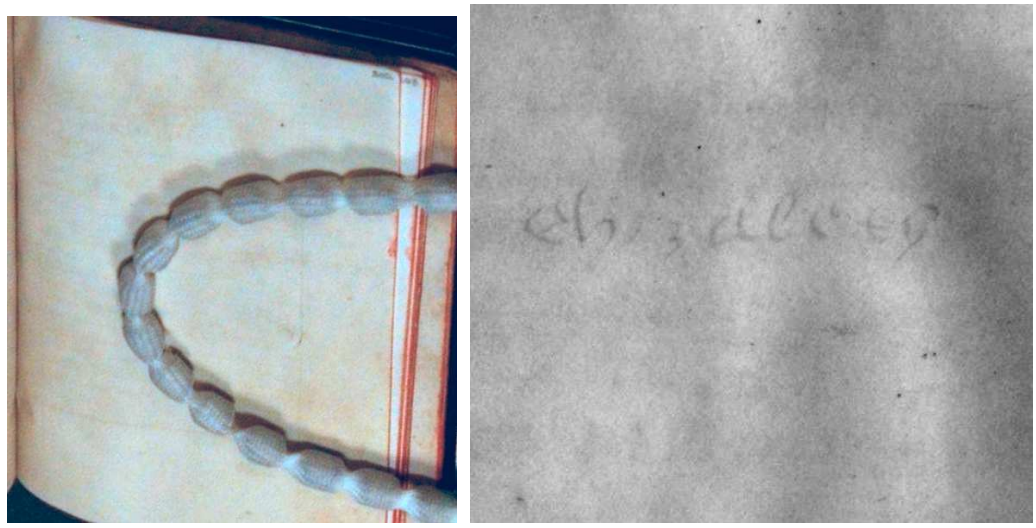

Fig. 4 (a) A colour photo of folio 202r of MS Lat. Liturg. g.1. from the Bodleian Library collection (courtesy of the Bodleian Libraries, University of Oxford); (b) near infrared image at $880 \mathrm{~nm}$ taken with a modified PRISMS (see Sec. 6) [91] of the top region of the page showing a clear signature [48]. 


\subsection{Colour rendering}

Multispectral imaging enables rendering of colour accurate images of paintings under any lighting conditions, unlike a normal tri-colour image which can only capture an accurate colour image under the specific illumination used at the time. The tri-stimulus values, $X, Y$ and $Z$ can be derived from the spectral reflectance through

$$
\begin{aligned}
X & =k \int_{0}^{\infty} R(\lambda) S(\lambda) \bar{x}(\lambda) d \lambda \\
Y & =k \int_{0}^{\infty} R(\lambda) S(\lambda) \bar{y}(\lambda) d \lambda \\
Z & =k \int_{0}^{\infty} R(\lambda) S(\lambda) \bar{z}(\lambda) d \lambda \\
k & =\frac{100}{\int_{0}^{\infty} S(\lambda) \bar{y}(\lambda) d \lambda}
\end{aligned}
$$

where $R$ is the spectral reflectance of the material, $S$ is the spectrum of the illumination normalised to 100 at $560 \mathrm{~nm}$ and $\bar{x}, \bar{y}, \bar{z}$ are the colour matching functions for the standard $2^{\circ}$ observer [52]. Once the spectral reflectance of a material has been determined, the colour of the material under illumination of any spectral density distribution can then be uniquely derived (Fig. 5). The tri-stimulus values are often converted to the CIE standard colour coordinates $L, a, b$ for a uniform colour-space [53] [52]. Discussions on the various CIE colour difference formula are given in [55] [54]. The colour difference $\Delta E$ is used to describe small colour differences and in conservation they are used to describe the fading of coloured material.

\subsection{Conservation monitoring}

Accurate recording of the spectral and therefore colour of paintings has been used for monitoring the change due to natural degradation, before and after transport, exhibition and conservation treatment as detailed in a review given by Martinez et al. [57]. In addition, due to the increased use of laser cleaning, multispectral imaging has been used for the on-line monitoring of laser cleaning of marbles [58][59], paper and parchment [60]. Both UV reflectance and UV-fluorescence images were found to be particularly useful in monitoring the cleaning process and investigating potential damages caused by laser cleaning.

One of the greatest problems with conservation of wall paintings and walls of historical structures is moisture. The water absorption bands around $1.4 \mu \mathrm{m}$ and $1.9 \mu \mathrm{m}$ can be used to monitor moisture content. Our group has used the PRISMS SWIR hyperspectral imager (see Sec. 6) to monitor moisture in walls using the $1.4 \mu \mathrm{m}$ band. 


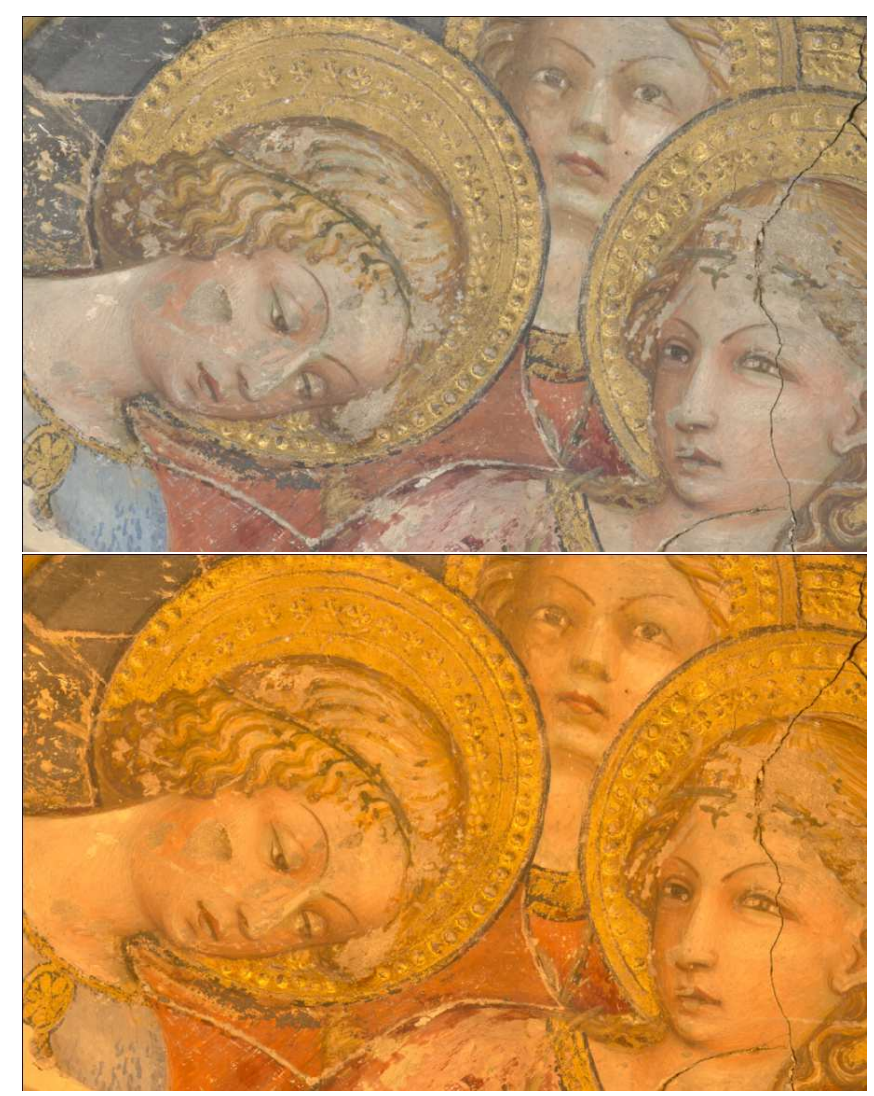

Fig. 5 Colour images derived from multispectral images taken with the upgraded VASARI system using CRISATEL set of interference filters [56][13] of Heads of Angels (NG 1842), an Italian fresco in the National Gallery, London: a) rendered in daylight (D65) and b) rendered in candle light (illuminant A) [56].

For monitoring purposes, good absolute calibration is necessary especially because the changes are likely to be very small. The earlier these changes can be detected, the more valuable the monitoring exercise is.

\subsection{Pigment identification}

Pigment identification using spectral reflectance has a long history (e.g. [61]), however, non-invasive spectral pigment identification in the visible and near infrared has not been met with a lot of enthusiasm in the conservation community so far. This is partly because the technique is perceived as not always yielding definitive identifications, especially where there is a complicated pigment mixture or that if the paint has degraded. In addition, for oil paintings, tiny paint samples can be taken from paintings provided that the sampling sites are near cracks and edges. Historically, 
this became possible when micro-chemical analysis became viable. Invasive chemical analyses always yield more chemically specific information, and often times multiple chemical analysis methods can be used on one sample. For paintings where multispectral imaging was first applied to, there has not been an overwhelming need for non-invasive pigment identification. This is, however, not the case for manuscripts where sampling is not possible. In particular, for illuminated manuscripts, non-invasive pigment identification is particularly valuable both for assisting conservation decisions and for historical studies [48].

One of the main obstacles with pigment identification using spectral reflectance is the lack of comprehensive databases of reference pigment and paint. A number of groups have their own reference pigment sets but few have a comprehensive set that has been systematically prepared and measured (e.g. [63] [62]). The only one that is publicly available is the CNRIFAC on-line database of spectral reflectance from $270 \mathrm{~nm}-1700 \mathrm{~nm}$ of various pigments in common binding media found on Western European paintings [63].

In the case of mixture of pigments, the identification of the pigments involves the 'unmixing' of the spectral components of the constituent pigments. Spectral unmixing in this context is very different from those in remote sensing [65][66]. In remote sensing, spectral unmixing is necessary mainly because the spatial resolution is relatively low due to the great distances involved and hence the spectral response from a pixel is a combination of various material that are spatially separated. Linear unmixing is therefore sufficient in such cases to separate out the spectra of the constituent material. For paint mixtures, the small pigment particles are uniformly dispersed in the binding medium and the reflectance spectrum of the paint mixture is not simply a linear mixture of the spectral reflectance of the paints of single pigments. Light transport in a medium is described by the radiative transfer (or transport) equations first developed in astrophysics to describe the effect of stellar atmosphere and interstellar medium on light propagation [71] [72]. Spectral unmixing for paint is best performed by modelling the physics of light transport in a turbid medium.

The Kubelka-Munk (KM) theory [73] [74] involving just two diffuse fluxes in the forward and backward direction which successfully describes the spectral reflectance of paint layers is a simple approximation to the full radiative transfer model. The main assumptions of the KM theory are 1) the paint layers or any turbid medium is homogeneous in the sense that the particle sizes are much smaller than the thickness of the layer; 2) the transverse extent of the layer is much greater than the the thickness; 3) the illumination is diffuse and the light propagation in both directions are uniformly diffuse. Limitations of the simple two-flux KM theory and comparisons with simple improvements are described in many papers (e.g. [75] $[76])$. The KM theory relates the diffuse reflectance $(\mathrm{R})$ of the layer without interfaces to the effective absorption $(K)$ and effective scattering coefficients 
(S) through

$$
R=\frac{1-R_{g}[a-b \operatorname{coth}(b S h)]}{a+b \operatorname{coth}(b S h)-R_{g}}
$$

where $a=(K+S) / S, b=\sqrt{a^{2}-1}, h$ is the layer thickness and $R_{g}$ is the reflectance of the substrate. In the limit of a paint layer with infinite optical thickness, the ratio between the absorption and scattering coefficients is related to the reflectance by

$$
\frac{K}{S}=\frac{\left(1-R_{\infty}\right)^{2}}{2 R_{\infty}}
$$

where $R_{\infty}$ is the spectral reflectance of a layer with infinite optical thickness. It should be noted that $K$ and $S$ do not always have a linear relation to concentration. KM theory assumes diffuse illumination and collection, however, in practise, measurements are often made with collimated normal illumination [76]. Saundersons correction [77] is normally applied to correct for the reflection at the air/paint and paint/air interfaces. The KM theory is so simple and successful that it is still being used in the paint industry to work out the mixing ratios of paints to match a given colour.

It is usually assumed that the effective absorption and scattering coefficients $(K$ and $S$ ) of a mixture is a linear combination of the constituent coefficients weighted by their concentrations. In the case where the pigments are all mixed with a highly scattering white paint, the final pigment mix can be described as a linear combination of $K / S$ since the white paint dominates the scattering [78]. The advantage of this simplification is that only one measurement of the spectral reflectance of a reference paint is necessary. Without this simplification, it is then necessary to measure $K(\lambda)$ and $S(\lambda)$ for each reference paint which usually involves either measurements of a semi-transparent paint layer over white and black substrates or measurements of the same paint with different thickness (always semi-transparent) over the same substrate.

If a mixture of different paint components can be modelled as a linear combination of $\mathrm{K} / \mathrm{S}$, then we can predict the reflectance of the paint mixture. This is sometimes referred to as the single constant KM theory. Since the aim here is to identify the pigments in a mixture, the concentrations of the single pigments were set as free parameters to find the best non-negative least squared fit of the predicted spectrum to the actual measured spectrum of the mixture for each combination of reference pigments [67] [79]. Liang et al. [67] showed that the method worked even for pigment mixtures without the addition of white pigments, except in cases where pigments with high absorption or very low scattering are involved. For pigments with high $K$ and low $S$, it is necessary to use the spectra of those pigments mixed with a white pigment to obtain a correct spectrum for the paint mixture. Figure 6 shows that a mixture of red earth and azurite paint can be correctly identified using the above method and the measured spectral reflectance of pure red earth paint and pure azurite paint. Figure 7 shows that a mixture of indigo and orpiment can be correctly identified using the spectral 


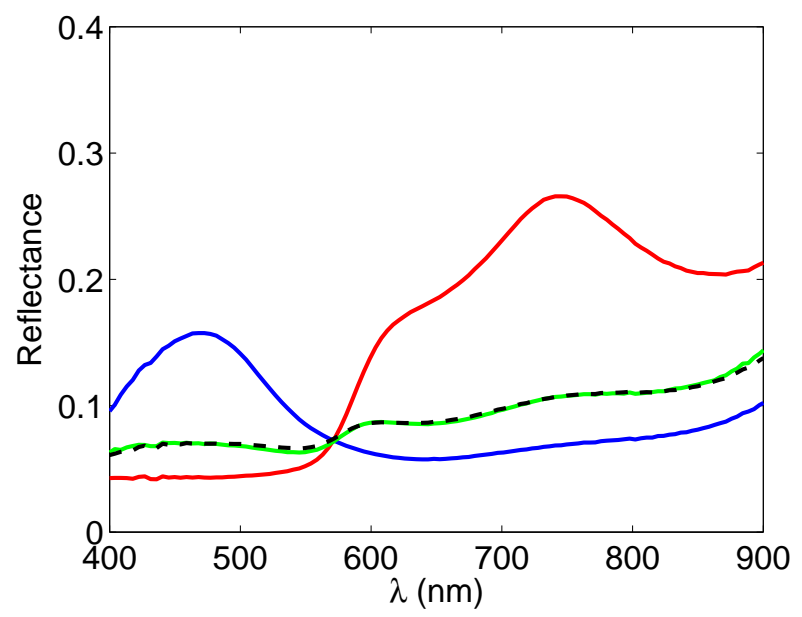

Fig. 6 The measured reflectance spectra of red earth in egg tempera (red curve) and azurite in egg tempera (blue curve) as well as their mixture (green curve). A spectrum of a mixture derived from the KM theory is shown in comparison (dashed black curve).

reflectance of indigo mixed with lead white and pure orpiment spectrum but not with pure indigo and pure orpiment spectrum since indigo has a very high absorption coefficient.

In contrast, Latour et al. [80] prepared a number of reference paint samples over black and white substrates and measured their $K$ and $S$ and assumed that the $K$ and $S$ of the mixture are linear combinations of their respective constituents to deduce the pigment mixture using a method similar to the above. This is sometimes referred to as the two constant KM theory.

It is important to know the effect of the various parameters associated with a paint that can affect pigment identification. The effect of particle size, concentration and types of binding medium on the spectral reflectance have been systematically studied by Feller [64] and Liang et al. [67]. Figure 8 shows that the pigment to medium ratio only affects the peak of the spectrum but not the general spectral shape. Figure 9 shows that the binding medium have by far the most dramatic effect on the spectral reflectance, changing even the ratios between the peaks, but the positions of the peaks remain unchanged. On the other hand, changes in particle size of pigments can result in peak shifts by as much as $20 \mathrm{~nm}$ as demonstrated in Fig. 10 for azurite and malachite with mean particle sizes between $30 \mu \mathrm{m}$ and $3 \mu \mathrm{m}$.

Finally, it is important to know how the effect of surface dirt and varnish might affect the measured spectral reflectance since historic paintings will have surface dirt and sometimes degraded yellowed varnish on top of the paint surface. Figure 11 shows that surface dirt acts like a neutral density filter and does not change the shape of the spectrum. Since many Western 

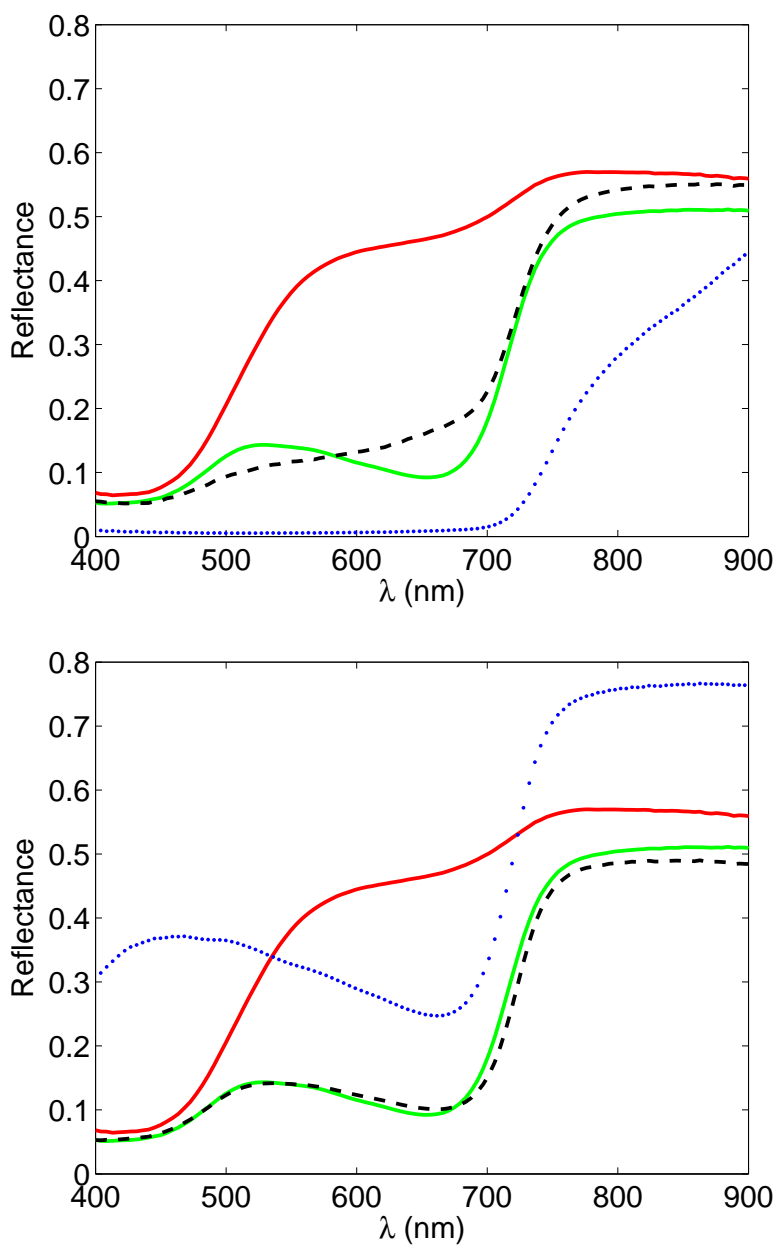

Fig. 7 a) The reflectance spectrum of indigo in linseed oil (blue curve) and orpiment in linseed oil (red curve) as well as a mixture of the two pigments in linseed oil (green curve). The spectrum of indigo and orpiment mixture is inconsistent with the spectrum of a mixture derived from KM theory using the single pigment paint spectra (dashed curve) b) The reflectance spectrum of the indigo and orpiment mixture predicted from KM theory (dashed curve) using the spectra of an indigo mixed with lead white in linseed oil (blue curve) and an orpiment in linseed oil spectrum (red curve) correctly predicts the spectrum of an indigo and orpiment mixture (green curve). 


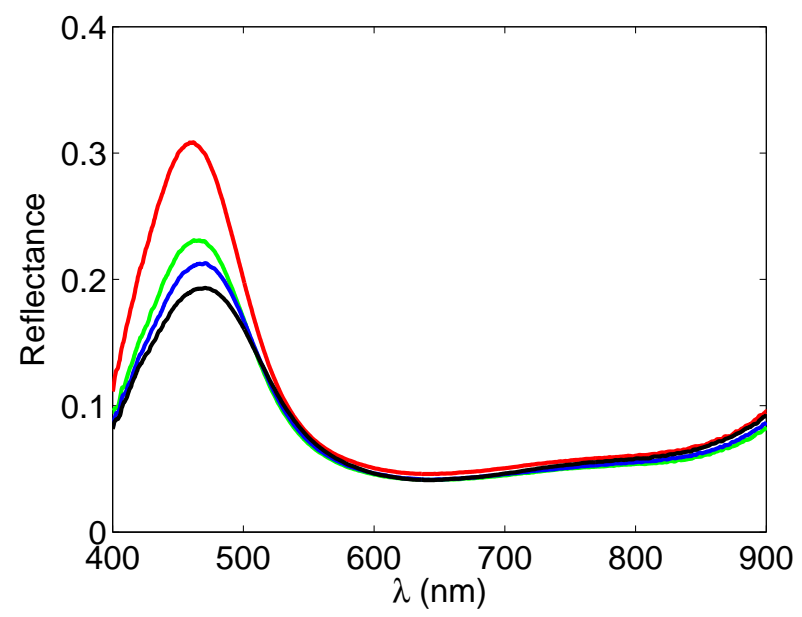

Fig. 8 The effect of pigment concentration in a binding medium on the reflectance spectra is shown for azurite in egg tempera. The concentrations are $89 \%$ (red curve), $78 \%$ (green curve), $67 \%$ (blue curve) and 56\% (black curve)

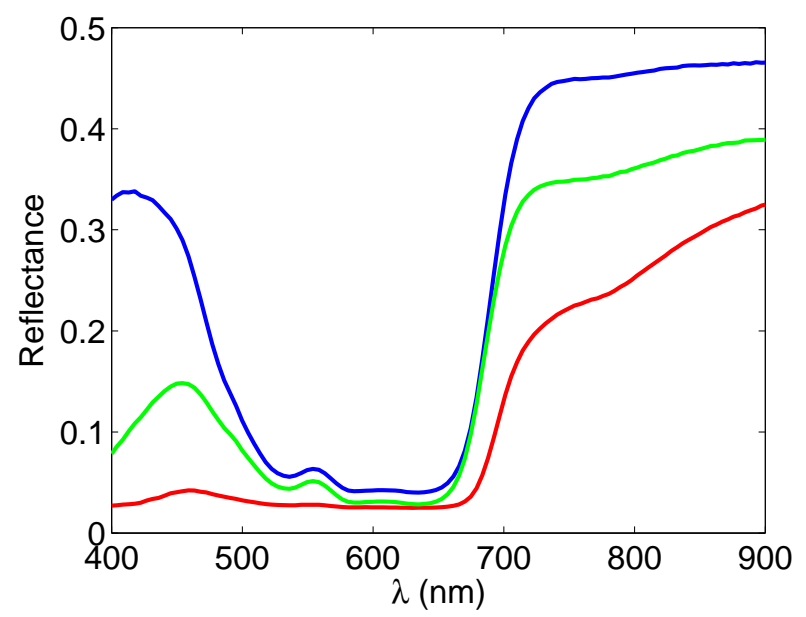

Fig. 9 The effect of the type of binding medium on the reflectance spectra is shown for smalt pigment in linseed oil (green curve), egg tempera (red curve) and acrylic (blue curve).

European paintings are varnished and old varnish tends to turn yellow, the effect of varnish is examined. Figure 12 shows that old varnish acts like a yellow filter which suppresses the blue reflectance, leaving the rest of the spectrum unchanged. The effect of relatively new varnish on spectral reflectance of paint has been studied in detail by Berns and de la Rie [69] and Elias et al. [68]. This study was also extended to thin layers of old varnish 

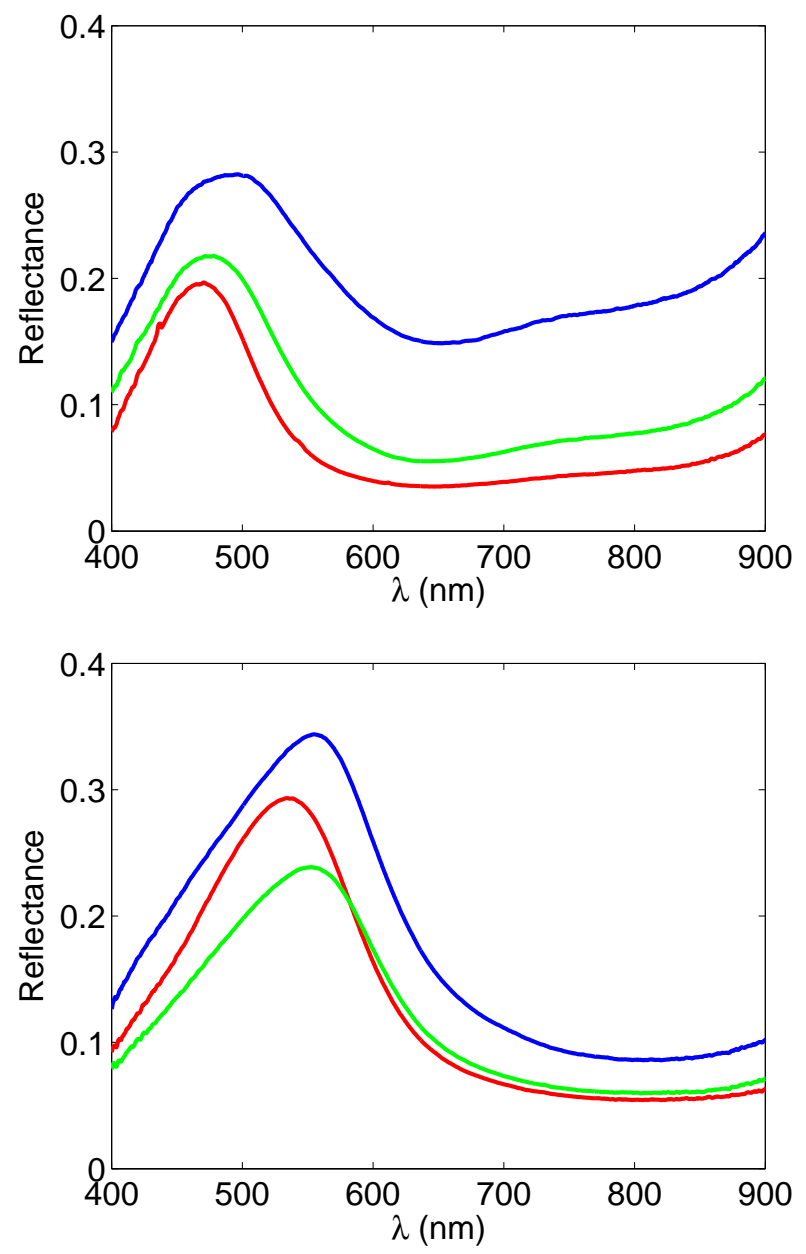

Fig. 10 The effect of particle size on the reflectance spectra for (a) azurite and (b) malachite in egg tempera (red for grade 1, green for grade 3 and blue for grade $5)$. Grade 1 is the largest $(\sim 30 \mu \mathrm{m})$ and grade 5 is the smallest $(\sim 3 \mu \mathrm{m})$.

in the longer wavelength spectral range where the effect of absorption due to the old yellowed varnish is not significant [70]. Figure 12 shows the significant absorption in the blue part of the reflectance spectrum of a paint measured through an old yellowed varnish.

Based on the knowledge of how the various factors such as particle size could affect the spectral reflectance and the method of identifying the pigment mixtures using the KM theory, Liang et al. [67] devised an algorithm to automatically identify pigments by fitting different combinations of reference paint spectra to an unknown spectrum. The best fit for each combination as well as single pigment spectra with and without white pigment 

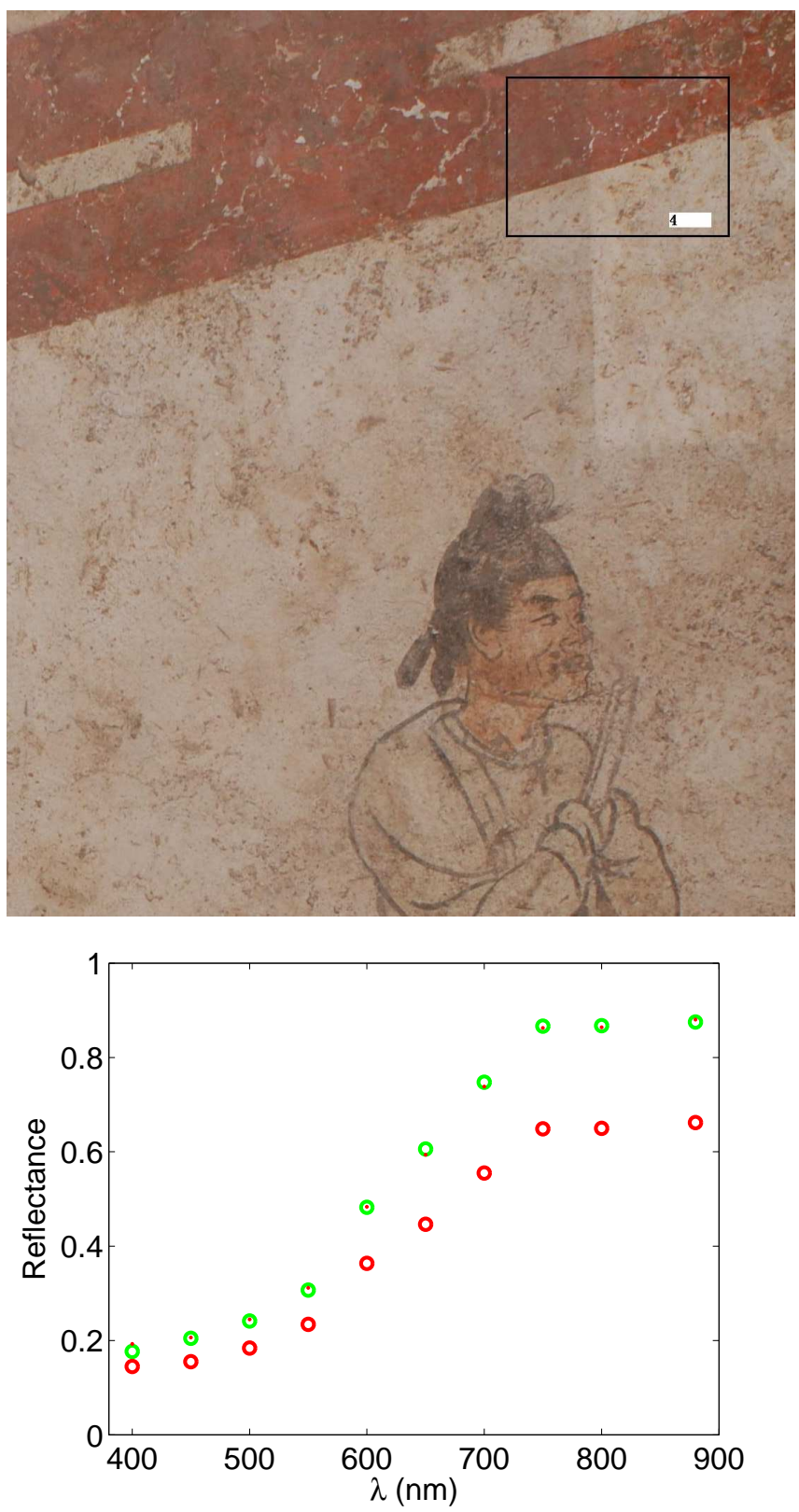

Fig. 11 The effect of surface dirt on the spectral reflectance. A colour photo of a region of a mural painting from the tomb of Prince Zhanghuai showing regions that have been test cleaned (copyright Shaanxi History Museum). The spectral reflectance of a red paint (from the region marked by a black box) in the area where it has been test cleaned (green circles) and a neighbouring area that has not been cleaned (red circles). The uncleaned reflectance spectrum is also scaled up by multiplying a constant (red dots) to show that the surface dirt does not change the spectral shape. 

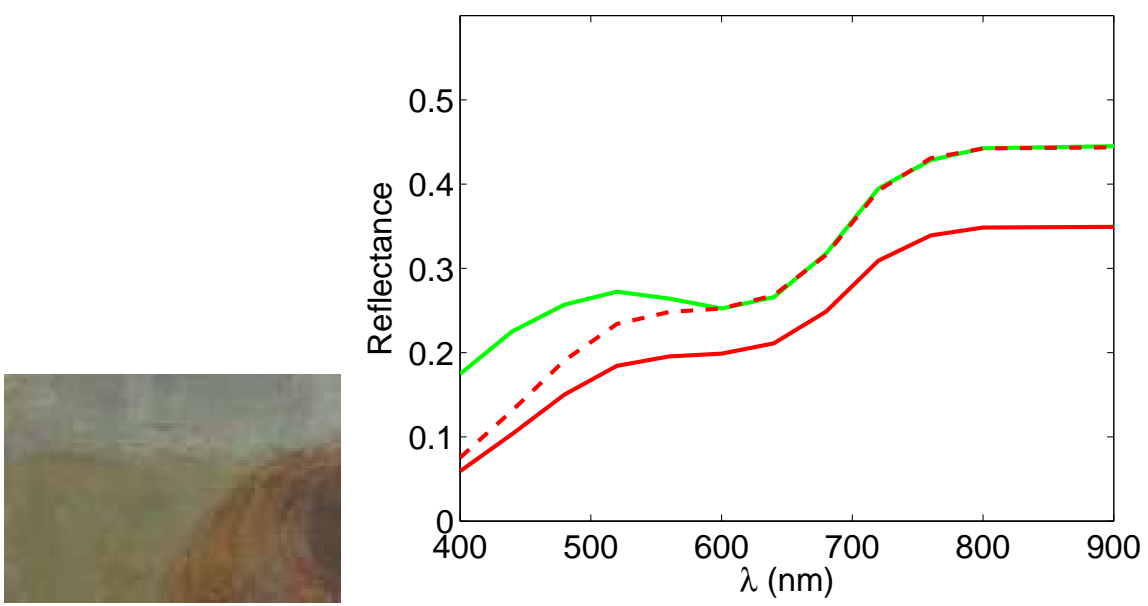

Fig. 12 The effect of yellowed old varnish on spectral reflectance. A colour image of a region of a test painting showing parts of the blue sky covered with yellowed varnish and parts that have the varnish cleaned off, as well as regions that were re-varnished with a new varnish. The spectrum from the yellowed varnish area (red curve) is also scaled up by multiplying a constant (red dashed curve) and compared with the unvarnish area (green curve) to show that the yellowed varnish absorbed strongly in the blue region.

mixtures are then cross-correlated with the unknown spectrum. The best identification is the one with the highest cross-correlation coefficient at zero offset. An extra $20 \mathrm{~nm}$ range around zero offset should be allowed to account for the potential shift in peaks due to particle size differences. Figure 13 shows an example of a spectral pigment identification using multispectral imaging of the painting in Fig. 3. The green paint was identified with a mixture of chrome yellow and Prussian blue. This identification was verified by an independent examination under the microscope of a small sample from the green region [83].

An alternative method to the KM theory, which solves the radiative transfer equation to predict the bi-directional reflectance of semi-transparent stratified paint and glaze layers, has been validated using known samples by Simonot et al. [81] and Latour et al. [82].

\subsection{Pigment mapping using multivariate statistics}

Multivariate statistics [84] is often used in analysing hyperspectral image cubes in remote sensing [4]. Similarly it is also used in chemometrics for spectral analysis. In spectral imaging of paintings, it is often used for rapid pigment mapping. For example, Baronti et al. [20] used principal component analysis to decompose the spectra into a number of orthogonal principal components. Similarly, Delaney et al. [32] used the standard remote sensing 


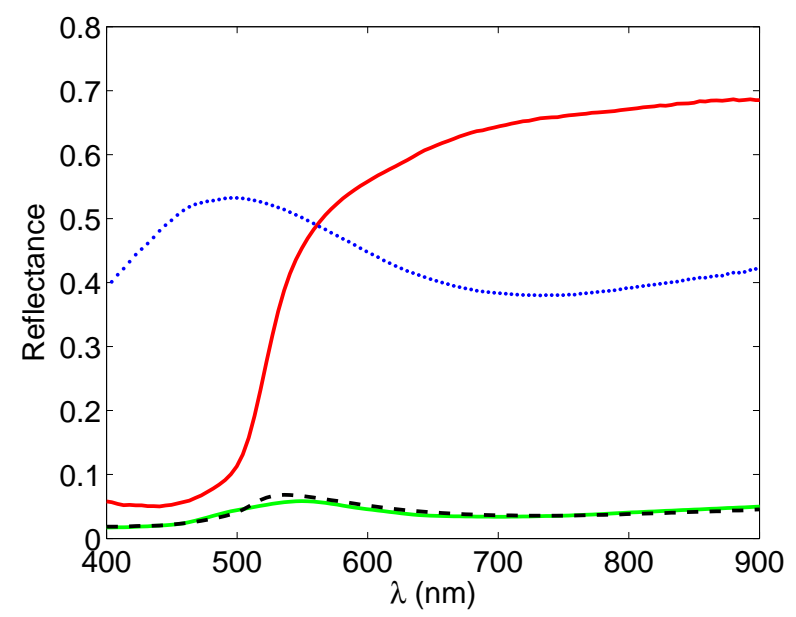

Fig. 13 The pigment mixture used in the green headdress of the virgin in Fig. 3 is identified with a mixture of chrome yellow and Prussian blue. The reflectance spectrum of a small area of the green headdress (green curve) captured with the PRISMS (Sec.6) multispectral camera is compared with the spectrum derived from KM theory (dashed curve) using a reference spectrum of chrome yellow in linseed oil (red curve) and Prussian blue and lead white in linseed oil (blue curve).

software ENVI to decompose the spectra into mutually independent endmembers. Most of these methods assume linear combination. Multivariate statistical methods are efficient at distinguishing different materials, however, the endmembers do not necessarily have any physical meaning and therefore cannot be compared to the spectra of reference material for the identification of material.

\subsection{UV-fluorescence}

UV-fluorescence has been used in conservation since the 1920s. It has been used to qualitatively identify old varnish and other organic material. Spectral imaging provides a means of quantitatively studying the fluorescence emission.

In spectral imaging systems where the filtering or wavelength selection is between the object and the detector, UV-fluorescence images can be readily obtained by using a low energy UV light source with a filter that restricts the incident light to a narrow spectral range around the intended excitation wavelength and blocking off radiation in the visible range of the spectrum where the material is likely to have fluorescence emission. The main difficulties of obtaining UV-fluorescence spectra is the radiometric calibration. It requires measurement of the spectral response of the entire imaging system. Early applications were semi-quantitative (e.g [35]), and later attempts used 
either indirect radiometric calibration [85] or measured directly the spectral response of the filter system and the CCD detector to derive the total spectral response of the system [86].

An extension of UV-fluorescence is laser induced fluorescence (LIF) which was first applied to aid pigment analysis [87]. LIF is a point-based technique where a spectrometer is used to obtain the fluorescence signal emitted at the laser illuminated spot. Recently, it has been used to map regions of interest at discrete spectral bands [88].

\section{Towards Remote Spectral Imaging}

There is always a trade-off between field of view and spatial resolution. For high resolution imaging of large objects such as large wall paintings, it is necessary to image a number of small areas at high resolution and then mosaic the images together. This usually requires imaging at close range, which means for objects at lofty heights, such as ceiling paintings, it is necessary to use scaffolding or mechanical lifting devices to move the imaging system close to the areas of interest [89] [90]. Recently a spectral imaging system specifically designed for remote imaging of wall paintings, PRISMS (Portable Remote Imaging System for Multispectral Scanning), has been developed in our group to overcome such difficulties by using a small amateur telescope [91] [67] [93].

PRISMS has a visible/NIR (400-880nm) multispectral imaging system as well as a short wave infrared (900-1700nm) hyperspectral imaging system (Fig. 14). The VIS/NIR system is a low budget system that consists of a filter wheel with 10 bandpass filters each with a bandwidth of $40 \mathrm{~nm}$ except for the one at $880 \mathrm{~nm}$ which has a bandwidth of $70 \mathrm{~nm}$ and a peltier cooled Jenoptics CCD camera [91]. The SWIR system consists of a Gooch \& Housego AOTF spectrograph and a Xenics InGaAs camera. The best spectral resolution of the AOTF is $10 \mathrm{~nm}$ and the central wavelength and bandwidth can be rapidly tuned automatically from a laptop [93]. A Meade ETX-90 telescope is used for high resolution imaging at distances greater than $3.5 \mathrm{~m}$ and lenses are used for close range imaging below $3.5 \mathrm{~m}$. Spatial resolution of tens of microns per pixel can be achieved for distances of $10 \mathrm{~m}$. The system also gathers 3D information, since the telescope mount is computer controlled with angular precisions of 1' to 3 ' and the distance can be determined to millimetre precision using the focus position for each scene. PRISMS is able to perform spectral imaging in the VIS/NIR and SWIR, as well as measuring the 3D surface texture of the object as a byproduct of the high spatial resolution imaging. The maximum distance range of operation depends on the intensity of the illumination. Figure 14 shows that the PRISMS system is flexible and can easily be adapted to close range imaging of manuscripts by swapping a lens with the telescope.

Laser induced fluorescence (LIF) has the clear advantage of having a narrow excitation spectral range and a high intensity, and therefore remote 

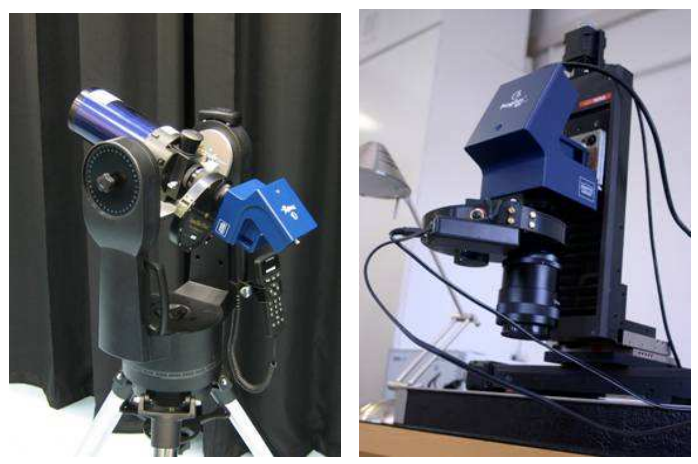

Fig. 14 The PRISMS system in the visible/NIR configuration for remote imaging at distance greater than $3.5 \mathrm{~m}$ and the modified PRISMS for scanning of manuscripts.

imaging potential. LIF has been used by a number of groups to remotely image buildings using a point by point scan to detect bio-degradation on stones [95] and frescoes [88] and detecting past conservation interventions and identifying stone types [94].

\section{Combined with other techniques}

The combination of multispectral and hyperspectral imaging in the visible and SWIR with other complementary non-invasive or micro-destructive techniques where possible, holds the promise of uncovering more detailed information about an object. The advantage of spectral imaging is its speed of capture and therefore it is possible to scan large areas.

\subsection{Combined with traditional micro-destructive techniques}

In cases where detailed chemical information is needed, for example the exact nature of the binding medium or pigment identification where the paint has significantly degraded, it is useful to complement the non-invasive imaging spectroscopy with traditional suite of micro-chemical analysis using tiny samples from the object if it is possible to do so (e.g. oil paintings). While the micro-destructive techniques give detailed chemical information from isolated tiny samples which might not be representative of a painting, the non-invasive spectral imaging technique can verify how representative the point is by comparing the spectrum at the point with other areas on the object [13] [92].

In addition, multispectral imaging combined with a microscope has been used to examine paint cross-sections to give spectral reflectance information in addition to the conventional colour information through visual examination of a sample cross-section under a microscope [96]. 


\subsection{Combined with laser scanning and photogrammetry}

Any object can be visually reconstructed if both its 3D spatial structure and spectral reflectance per point are characterised. Even seemingly 2D objects like paintings have 3D texture information. For wall painting, 3D mapping is particularly important because of the surface roughness and the continuous motif that covers large areas of the interior of an architectural structure. There has been a number of projects where multispectral imaging has been combined with 3D capture using laser scanning and photogrametry [98] [97].

\subsection{Combined with X-Ray Fluorescence (XRF)}

X-ray fluorescence is a non-invasive, in situ point analysis technique (in the portable versions) for the identification of elements with atomic number higher than sodium. It complements the large area scans of imaging spectroscopy. While XRF is an elemental analysis technique, imaging spectroscopy is sensitive to the chemical composition of the compound (e.g. the paint mixture). As described in Sect. 5.4, the pigment, the binding medium, the pigment particle size, concentration can all affect the spectral reflectance of a paint. On the other hand, XRF is able to detect not only the main elemental composition but also trace elements which can be useful to study the source of pigments. While the complementary nature of the two noninvasive techniques have been known for a long time, few studies have used the two in combination [99] [21] [32] [100].

\subsection{Combined with Optical Coherence Tomography (OCT)}

OCT is a relatively new non-invasive technique for the imaging of subsurface microstructure of transparent and semi-transparent material in the near infrared (see review on OCT in this special issue). OCT gives not only the subsurface layer structures but also reveals the scattering and absorption properties of the material [62]. OCT complements spectral imaging in the identification of the material and providing information on the surface topology and subsurface layer structure. A study combining OCT and multispectral imaging was conducted by our group on an easel painting [67] and a wall painting [101]. Combined spectral imaging and OCT images of virtual cross-sections of paint layers has the potential to match the information content given by microscopic examinations of real sample cross-sections.

\section{Future}

Hardware development of multispectral and hyperspectral imaging has reached maturity with many commercially available instruments. However, it is important to realise that the right instrument with the appropriate software 
must be chosen for a specific application. An instrument designed for microscopic hyperspectral imaging may not be suitable for remote spectral imaging without hardware/software modification. It would be useful to extend the spectral imaging capabilities to the mid-IR, the wavelength range traditionally used for FTIR where more chemically specific information can be obtained. Some very recent progress has been made in this direction [102]. Given the heterogeneous nature of heritage applications, it is best to build modular systems that are versatile and flexible.

Future research should concentrate on further development of material identification methods using spectra collected with hyperspectral imaging systems that cover a broad wavelength range. One critical ingredient is the development of comprehensive databases of reference material. More efficient methods of presenting the data are needed, since spectral imaging is soon to become one of the tools routinely used by the heritage community for the recording and the scientific examination of artefacts. Most of the applications so far have concentrated on paintings and manuscripts. Applications on other types of object, especially intact objects where invasive methods are not allowed, should be further explored. Closer collaborations with practitioners from the heritage community is needed for spectral imaging to solve real problems and hence further demonstrate its relevance to art conservation and archaeology.

Acknowledgements Contributions from past and current research fellows, students and technicians at Nottingham Trent University are gratefully acknowledged: Rebecca Lange, Simon Godber, Tom Vajzovic, Kafing Keita, Andrei Lucian, Borislava Peric, David Parker and Sammy Cheung. Special thanks to Rebecca Lange for making last minute illustrations. I would like to thank my collaborators John Cupitt, Helen Howard, Sophie Julien-Lees, Sarah Neate, Chris Pannell, David Saunders, Marika Spring, Jane Spooner, Jon Ward and Qunxi Zhang for valuable contributions to the various spectral imaging projects. Some of the work presented here was funded by UK Engineering and Physical Sciences Research Council (EPSRC) grant (EP/E016227/1), EU FP5 supported CRISATEL project (IST1999-20163), Royal Society Research Grant (2005-2006), Leverhulme Trust Project Grant (2006-2009) and a Stimulating Innovation for Success grant from Nottingham Trent University (2006-2007).

\section{References}

1. A. Aldrovandi, D. Bertani, M. Cetica, M. Matteini, A. Moles, P. Poggi, P. Tiano, Studies in Conservation 33, 154 (1988)

2. M. Hain, J. Bartl, V. Jacko, Measurement Science Review 3, 9 (2003)

3. A. Goetz, G. Vane, J. Solomon, B. Rock, Science 228, 1147 (1985)

4. H. Grahn, P. Geladi (editors), Techniques and Applications of Hyperspectral Image Analysis, John Wiley \& Sons, 2007

5. P. Yuen, M. Richardson, The Imaging Science Journal 58, 241 (2010)

6. C. Fischer, J. Kakoulli, Reviews in Conservation 73 (2006) 
7. M. Kubik, in Physical Techniques in the Study of Art, Archaeology and Cultural Heritage, Vol. II, Amsterdam 2007, p.199

8. A. Burmester, J. Cupitt, H. Derrien, N. Dessipris, A. Hamber, K. Martinez, M. Müller, D. Saunders, in 3rd International Conference on Non Destructive Testing, Microanalytical Methods and Environmental Evaluation for Study and Conservation of Works of Art, Rome 1993, p.201

9. D. Saunders, J. Cupitt, National Gallery Technical Bulletin, 14, 72 (1993)

10. K. Martinez, J. Cupitt, D. Saunders, Proc. SPIE 1901, 25 (1993)

11. C. Lahanier, G. Alquié, P. Cotte, C. Christofides, C. de Deyne, R. Pillay, D. Saunders, and F. Schmitt, in International Council of Museums, Committee for Conservation (ICOM-CC), 13th Triennial Meeting Rio de Janeiro, James \& James London 2002, p. 295

12. A. Ribés, H. Brettel, F. Schmitt, H. Liang, J. Cupitt, D. Saunders, in Proceedings of the PICS Conference The Digital Photography Conference, IS\&T, Springfield, VA 2003, p. 215

13. H. Liang, D. Saunders, J. Cupitt, Journal of Imaging Science \& Technology 49, $551(2005)$

14. G. Thomson, The Museum Environment, 2nd edition (Butterworth Heine Series in Conservation and Museology, Oxford 1986)

15. D. Saunders, J. Kirby, in ICOM Committee for Conservation 11th Triennial Meeting Edinburgh, James \& James London 1996, p. 87

16. A. Lerwill, J. Townsend, H. Liang, J. Thomas, S. Hackney, E-Preservation Science 5, 17 (2008)

17. H. Liang, R. Lange, A. Lucian, P. Hyndes, J. Townsend, S. Hackney, in International Council of Museums, Committee for Conservation (ICOM-CC), 16th Triennial Conference Lison, Critério Produção Gráfica Lda., Almada 2011.

18. M. Klein, B. Aalderink, R. Padoan, G. de Bruin, T. Steemers, Sensors 8, 4476 (2008)

19. L. Fauch, E. Nippolainen, V. Teplov, A. A. Kamshilin, Optics Express 18, $23394(2010)$

20. S. Baronti, A. Casini, F. Lotti, S. Porcinai, Applied Optics 37, 1299 (1998)

21. J. K. Delaney, E. Walmsley, B. H. Berrie, C. F. Fletcher, in Sackler NAS Colloquium Scientific Examination of Art: Modern Techniques in Conservation and Analysis, National Academy of Science 2005, p120

22. N. Gat, Proc. SPIE 4056, 50 (2000)

23. J. Hardeberg, F. Schmitt, H. Brettel, Opt. Eng. 41, 2532 (2002)

24. R. Berns, L. Taplin, F. Imai, E. Day, D. Day, Studies in Conservation 50, 253 (2005)

25. J. Mansfield, M. Attas, C. Majzels, C. Collins, E. Cloutis, H. Mantsch, Vibrational Spectroscopy 28, 59 (2002)

26. N. Gupta, Proc. SPIE 6940, 69401P-1 (2008)

27. C. Stedham, M. Draper, J. Ward. E. Wachman, C. Pannell, Proc. SPIE 6889, $68891 \mathrm{C}(2008)$

28. D. N. Stratis, K. L. Eland, J. C. Carter, S. J. Tomlinson, S. M. Angel, Applied Spectroscopy 55, 999 (2001)

29. J. Bland-Hawthorn, in Encyclopaedia of Astronomy 83 Astrophysics, MacMillan and Institute of Physics Publishing, 2000

30. W. Marinelli, C. Gittins, A. Gelb, B. Green, Applied Optics 38, 2594 (1999) 31. A. Casini, M. Bacci, C. Cucci, F. Lotti, S. Porcinai, M. Picollo, B. Radicati, M. Poggesi, L. Stefani, Proc. SPIE 5857, 177 (2005) 
32. J. K. Delaney, J. G. Zeibel, M. Thoury, R. Littleton, M. Palmer, K. M. Morales, E. R. de la Rie, A. Hoenigswald, Applied Spectroscopy 64, 584 (2010)

33. M. Picollo, M. Bacci, A. Casini, F. Lotti, M. Poggesi, L. Stefani, in Conservation Science 200\%, Milan 2007, Archetype Publications, p162

34. J. Kerekes, J. Schott, in Hyperspectral Data Exploitation - Theory and Applications (Wiley, 2007) p20

35. A. Casini, F. Lotti, M. Picollo, L. Stefani, A. Aldrovandi, in Conservation Science 2002, Edinburgh 2002, p248

36. P. Carcagni, A.D. Patria, R. Fontana, M. Greco, M. Mastroianni, M. Materazzi, E. Pompaloni, L. Pezzati, Optics and lasers in Engineering 45, 360 (2007)

37. C. Bonifazzi, P. Carcagni, R. Fantana, M. Greco, M. Mastroianni, M. Materazzi, E. Pampaloni, L. Pezzati, D. Bencini, J. Opt. A: Pure Appl. Opt. 10, $064011(2008)$

38. C. Daffara, E. Pampaloni, L. Pezzati, M. Barucci, R. Fontanna, Accounts of Chemical Research 43(6), 847 (2010)

39. J. M. Eichenholz, N. Barnett, Y. Juang, D. Fish, S. Spano, E. Lindsley, D. L. Farkas, Proc. SPIE 7568, 75681L (2010)

40. R. S. Berns, L. A. Taplin, M. Nezamabadi, M. Mohammadi, Y. Zhao, in ICOM Committee for Conservation, 14th Triennial Meeting The Hague, James \&James London 2005, p.743

41. S. Howell, Handbook of CCD Astronomy (Cambridge University Press, 2nd edition, 2006)

42. B. Zitova, J. Flusser, Image and Vision Computing 21, 977 (2003)

43. J. Cupitt, K. Martinez, Proc. SPIE 1663, 19 (1996)

44. D. Saunders, J. Cupitt, National Gallery Technical Bulletin, 16, 61 (1995)

45. R. Billinge, J. Cupitt, N. Dessipris, D. Saunders, Studies in Conservation 38, 92, 1993

46. J. van Asperen de Boer, Studies in Conservation 14, 96 (1969)

47. C. Balas, V. Papadakis, N. Papadakis, A. Papadakis, E. Vazgiouraki, G. Themelis, Journal of Cultural Heritage 4, 330 (2003)

48. H. Liang, in The Technical Study of Books and Manuscripts as Artefacts research questions and analytical solutions, Archaeopress Oxford, 2011, p55

49. D.J. Walvoord, R.L. Easton Jr., IEEE Signal Processing Magazine 25(4),100 (2008)

50. K. Knox, R. Easton, W. Christens-Barry in 16th European Signal Processing Conference (EUSIPCO 2008), (Lausanne 2008) on-line publication

51. S.J. Kim, F. Deng, M.S. Brown, Pattern Recognition 44, 1461 (2011)

52. CIE Colorimetry, 3rd Ed. CIE Publication 015:2004, Vienna: Central Bureau of the CIE (2004)

53. K. McLaren, Journal of the Society of Dyers and Colourists 92, 338 (1976)

54. CIE Technical report: Improvement to Industrial Colour-difference Evaluation, CIE Publication 142-2001, Vienna: Central Bureau of the CIE (2001)

55. M. R. Luo, G. Cui, B. Rigg, Color Research and Application, 26(5), 340 (2001)

56. H. Liang, D. Saunders, J. Cupitt, C. Lahanier, in Conservation of Ancient Sites on the Silk Road: The Second International Conference on the Conservation of Grotto Sites, DunHuang 2004, (Getty Publications 2010) p.267

57. K. Martinez, J. Cupitt, D. Saunders, R. Pillay, Proc. IEEE 90(1), 28 (2002)

58. P. Pouli, V. Zafiropulos, C. Balas, Y. Doganis, A. Galanos, Journal of Cultural Heritage 4, 338 (2003) 
59. V. Papadakis, A. Loukaiti, P. Pouli, Journal of Cultural Heritage 11, 325 (2010)

60. W. Kautek, S. Pentzien, D. Müller, K. Troschke, R. Teule, Proc. SPIE 4402, $130(2001)$

61. M. Bacci, Sensors and Actuators 29, 190 (1995)

62. H. Liang, B. Peric, M. Hughes, A. Podoleanu, M. Spring, D. Saunders, Proc. SPIE 6618, 661805 (2007)

63. CNR-IFAC, Fibre Optics Reflectance Spectra Database of Pictorial Materials in the 270 to $1700 \mathrm{~nm}$ Range, http://fors.ifac.cnr.it/

64. R. Johnston-Feller, in Color Science in the Examination of Museum Objects - Nondestructive Procedures (Getty Publications, 2001)

65. J. Bowles, D. Gillis, in Hyperspectral Data Exploitation - Theory and Applications (Wiley, 2007) p77

66. J. Zhang, B. Rivard, A. Sanchez-Azofeifa, Remote Sensing of Environment, 95, $57(2005)$

67. H. liang, K. Keita, B. Peric, T. Vajzovic, in Proc. OSAV2008, The 2nd Int. Topical Meeting on Optical Sensing and Artificial Vision St. Petersburg, 2008, p33

68. M. Elias, L. Simonot, M. Menu, Optics Communications 191, 1 (2001)

69. R. S. Berns, E. R. de la Rie, in ICOM Committee for Conservation, 13th Triennial Meeting Rio de Janeiro (James \& James London, 2002), p.211

70. M. Elias, L. Simonot, M. Thoury, J. Frigerio, Optics Communications 231, $25,(2004)$

71. A. Schuster, Astrophysical Journal 21, 1 (1905)

72. S. Chandrasekhar, Radiative Transfer, Dover, New York, 1950

73. P. Kubelka, F. Munk, Z. Tech. Phys. 12, 593 (1931)

74. P. Kubelka, J. Opt. Soc. Am. 38, 448 (1948)

75. J. Nobbs, Rev. Prog. Coloration 15, 66 (1985)

76. W. Vargas, G. Niklasson, Applied Optics 36, 5580 (1997)

77. J. L. Saunderson, J. Opt. Soc. Am. 32, 727 (1942)

78. R. Berns, J. Kreuger, M. Swicklik, Studies in Conservation 47, 46 (2002)

79. Y. Zhao, R. Berns, L. Taplin, J. Coddington, Proc. SPIE 6810, 681007 (2008)

80. G. Latour, M. Elias, J. Frigerio, Applied Spectroscopy 63, 604 (2009)

81. L. Simonot, M. Elias, E. Charron, Applied Optics 43, 2580 (2004)

82. G. Latour, M. Elias, J. M. Frigerio, J. Opt. Soc. Am. A 24, 3045 (2007)

83. A. Roy, G. Mancini, National Gallery Technical Bulletin 31, 64 (2010)

84. M. Baxter, Statistics in Archaeology (Arnold Applications of Statistics, London, 2003)

85. A. Pelagotti, L. Pezzati, A. Piva, A. Mastio, in 14th European Signal Processing Conference, Florence, Italy 2006

86. D. Comelli, G. Valentini, A. Nevin, A. Farina, L. Toniolo, R. Cubeddu, Review of Scientific Instruments 79, 086112 (2008)

87. A. Anglos, M. Solomidou, I. Zergioti, V. Zafiropulos, T. Papazoglou, C. Fotakis, Applied Spectroscopy 50, p1331 (1996)

88. F. Colao, R. Fantoni, L. Fiorani, A. Palucci, I. Gomoiu, Journal of Optoelectronics and Advanced Materials 7, 3197 (2005)

89. E. Lange, British Museum Occasional Paper 114, 1 (1996)

90. Macdonald, L. Proc. SPIE 6069, 60690I (2006)

91. H. Liang, K. Keita, T., Vajzovic, Proc. SPIE 6618, 661815 (2007)

92. H. Liang, K. Keita, T. Vajzovic, in ICOM Committee for Conservation, 15th

Triennial Conference New Delhi, Allied Publishing, 2008, p353 
93. H. Liang, K. Keita, C. Pannell, J. Ward, in IX CONGRESO NACIONAL DEL COLOR, Alicante, Spain 2010, p189

94. L. Palombi, D. Lognoli, V. Raimondi, G. Cecchi, J. Hällström, K. Barup, C. Conti, R. Grönlund, A. Johansson, S. Svanberg, Optics Express 16, 6794 (2008)

95. D. Lognoli, G. Lamenti, L. Pantani, D. Tirelli, P. Tiano, L. Tomaselli, Applied Optics 41, $1780(2002)$

96. J. van der Weerd, M. van Veen, R. Heeren, J. Boon, Anal. Chem. 75, 716 (2003)

97. N. Brusco, S. Capeleto, M. Fedel, A. Paviotti, L. Poletto, G. M. Cortelazzo, G. Tondello, Machine Vision and Application 17, 373 (2006)

98. L. Barazzetti, F. Remondino, M. Scaioni, M. Lo Brutto, A. Rizzi, R. Brumana, in International Archives of Photogrammetry, Remote Sensing and Spatial Information Science, Vol. XXXVIII, Part 5, Commission V Symposium, 2010, p62

99. A. Casini, F. Lotti, M. Picollo, L. Stefani, E. Buzzegoli, Studies in Conservation 44, 39 (1999)

100. R. Lange, H. Liang, Q. Zhang, Proc. SPIE 8084, 80840Y (2011)

101. H. Liang, R. Lange, H. Howard, J. Spooner, Proc. SPIE 8084, 80840F (2011)

102. C. Daffara, L. Pezzati, D. Ambrosini, D. Paoletti, R. Di Biase, P. I. Mariotti, C. Frosinini, Proc. SPIE 8084, 808406 (2011) 